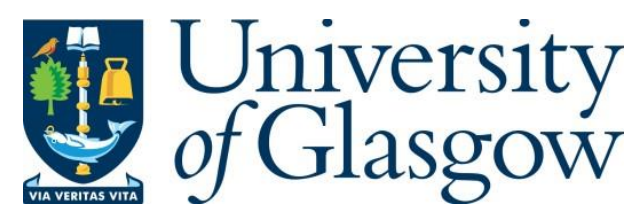

Simon, G., Andrade, M.A.B., Roolvink, D., Cormack, P.A.G., Riehle, M.O. and Bernassau, A.L. (2016) A Deterministic Method for Particle Sorting by Dynamic Acoustic Fields. In: 2016 IEEE International Ultrasonics Symposium (IUS), Tours, France, 18-21 Sep 2016, ISBN 9781467398978.

There may be differences between this version and the published version. You are advised to consult the publisher's version if you wish to cite from it.

http://eprints.gla.ac.uk/151588/

Deposited on: 13 November 2017

Enlighten - Research publications by members of the University of Glasgow http://eprints.gla.ac.uk 


\title{
A Deterministic Method for Particle Sorting by Dynamic Acoustic Fields
}

\author{
G. Simon 1 , M. A. B. Andrade 2 , D. Roolvink ${ }^{3}$, P. A. G. Cormack ${ }^{3}$, M. O. Riehle ${ }^{4}$, A. L. Bernassau ${ }^{1 *}$ \\ ${ }^{1}$ School of Engineering and Physical Sciences, Heriot-Watt University, Edinburgh, United Kingdom \\ ${ }^{2}$ Institute of Physics, University of São Paulo, Brazil \\ ${ }^{3}$ WestCHEM, Department of Pure and Applied Chemistry, University of Strathclyde, Thomas Graham Building, 295 Cathedral \\ Street, Glasgow, G1 1XL, United Kingdom \\ ${ }^{4}$ Centre for Cell Engineering, Institute for Molecular, Cell and Systems Biology, CMVLS, University of Glasgow, Glasgow, \\ United Kingdom
}

\begin{abstract}
This paper investigates the dynamic acoustic sorting method from a theoretical perspective. Analytical equations of motion and numerical simulations have been used to optimize parameters for sorting various particle mixtures. The effect of changes in field parameters as frequency or voltage on efficient sorting has been investigated. Experiments have been carried out to verify theoretical findings.
\end{abstract}

Keywords-acoustic particle sorting; acoustic particle manipulation; dynamic acoustic fields; acoustic tweezing; acoustic radiation force; phase modulation

\section{INTRODUCTION}

Cell separation is crucial in many research areas including clinical sample preparation, regenerative medicine, stem cell research and improved food safety [1]. The applicable methods for cell separation comprise electric, dielectric, magnetic, hydrodynamic and acoustic techniques [2-4]; however acoustic techniques have an advantage as they offer contactless and scalable sorting with negligible adverse effects on particles [5]. A wide range of approaches which exploit differences in particle migration times has been presented previously. These comprise a half-wavelength resonator structure, a frequency sweeping device, and the dynamic acoustic fields approach (DAF) [6]. The feasibility of our DAF method has been investigated earlier for sorting particles by size. In this paper, a detailed and predictive theoretical background for the DAF method is presented. The effects of density, compressibility of media and excitation frequency on the separation efficiency and purity of the sorted sample are investigated.

\section{METHOD}

When two opposing ultrasonic transducers are activated by the same sinusoidal signal an acoustic standing wave pattern is formed. The spherical particles suspended in the fluid media between transducers scatter the acoustic field and give rise to the primary acoustic radiation force:

$$
F_{r}=V_{r} k E_{a c} \Phi \sin (2 k x)
$$

where $V_{r}, k, E_{a c}$ and $\Phi$ are the volume of the particle, wavenumber, acoustic energy density and acoustic contrast factor, respectively. The acoustic contrast factor depends on the density $(\rho)$ and compressibility $(\kappa)$ of particle (subscript $p$ ) and media (subscript $m$ ) as

$$
\Phi=\frac{5 \rho_{p} / \rho_{m}-2}{2 \rho_{p} / \rho_{m}+1}-\frac{\kappa_{p}}{\kappa_{m}}
$$

Therefore the acoustic radiation force scales with the cube of the radius of the particle, and also has a weak dependence on the density. Based on the particle parameters they can either have negative or positive acoustic contrast factor. Particles with positive contrast factor are pushed towards the pressure nodes by the acoustic radiation force, while particles with negative contrast factor agglomerate along the antinodes. This can be readily utilized for binary fractionation [2]. When the acoustic energy density is appropriately chosen for the flow rate in the microchannel, separation due to the difference in mobility of particles can be achieved. These free flow fractionation devices have also been extensively investigated in the literature [3, 4].

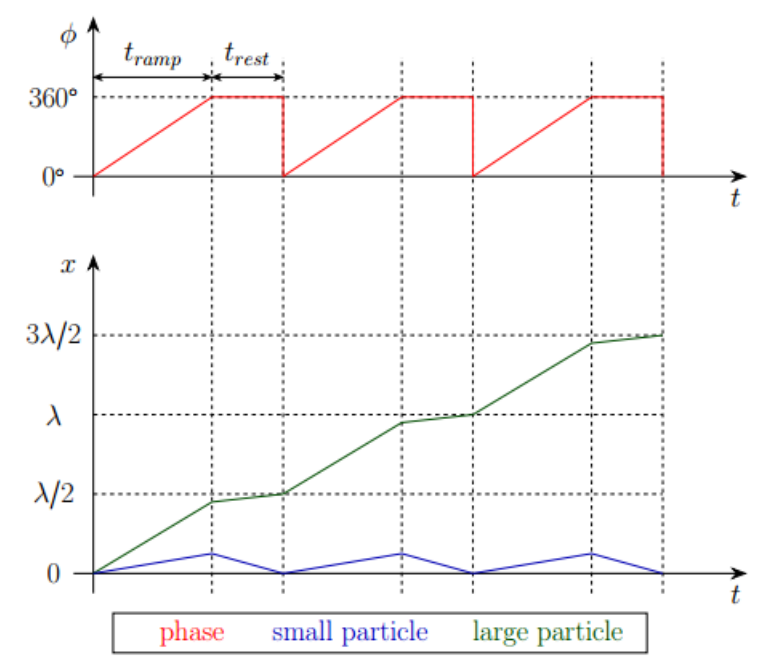

Fig. 1. The dynamic acoustic fields method. The top graph shows the phase shift pattern, the bottom graph shows the induced particle displacement

Our method for sorting particles utilizes a dynamic acoustic fields (DAF), where the phase of one of the transducers is shifted

*corresponding author, A.Bernassau@hw.ac.uk 
temporally resulting in a lateral displacement of the acoustic pattern and pressure nodes. When the movement of the nodes is slow enough, the trapping force on particles is large enough to drag them with the pattern, however smaller particles will not be able to follow. This difference in behavior allows for sorting. The phase pattern consists of two regions: firstly, it is linearly shifted from $0^{\circ}$ to $360^{\circ}$, and the time required for this is called the ramp time. Secondly the phase is kept steady to allow the particles to stabilize their position (Fig. 1).

To be able to design experiments and verify experimental results an analytical solution for the differential equation of motion shall be presented for our dynamic sorting method. As during the ramping the radiation force changes spatially, it has a different form to (1). Denoting the start of phase shift as $t_{s}$, defining the rate of phase shift as

$$
s=\frac{2 \pi}{t_{\text {ramp }}}
$$

and collecting all parameters of the radiation force into the single variable $c_{\mathrm{rad}}$, the force has the form

$$
F_{r}=-c_{r a d} \sin \left(2 k x-s\left(t-t_{s}\right)\right)
$$

Newton's second law for the forces acting horizontally (the radiation force and the Stokes' drag force) takes the form

$$
m \frac{d^{2} x}{d t^{2}}-F_{v}-F_{r}=0
$$

Neglecting the acceleration term since in microfluidics inertial effects are minuscule [7] we arrive at

$$
c_{v i s c} \frac{d x}{d t}+c_{\text {rad }} \sin \left(2 k x-s\left(t-t_{s}\right)\right)
$$

and substitution of the argument of the sin with a new variable gives the solution of the form

$$
x(t)=\frac{s\left(t-t_{s}\right)}{2 k}-\frac{1}{k} \tan ^{-1}\left(\frac{\gamma-Q \tan \left(\left(c_{i} Q-t Q\right) / 2\right)}{s}\right)
$$

where $\gamma=2 k c_{\text {rad }} / c_{\text {visc }}, Q=\left(s^{2}-\gamma^{2}\right)^{1 / 2}$ and $\tan ^{-1}()$ is the inverse tangent function normalized to $[0, \pi)$. The constant $c_{i}$ can be determined from initial conditions, and can be complex such as $Q$.

For the range where the phase is kept constant at $360^{\circ}$, the equation presented in [7] has been used. The only difference is the presence of a minus sign due to the different definition of the origin:

$$
x(t)=\frac{1}{k} \tan ^{-1}\left(c_{i} \exp (-\gamma t)\right)
$$

and $\gamma$ is defined as before. Therefore, the position of any particle can be determined theoretically using these equations.

The validity of these equations for particle trajectories has been verified by comparing results obtained from direct numerical solution of the differential equation of motion. Both approaches were implemented in MATLAB, the new method being significantly faster allowing for algorithms for parameter optimization.

\section{RESULTS AND DISCUSSION}

\section{A. Parameters Optimisation with DAF}

For a given set of particles separation can be achieved by choosing the appropriate $t_{\text {ramp }}$ and $t_{\text {rest }}$ time. Since the analytical equations for position are complex, a numerical approximation can be used instead for ideal parameter determination. An example is given for 10 and $15 \mu \mathrm{m}$ polystyrene particles in Fig. 2.

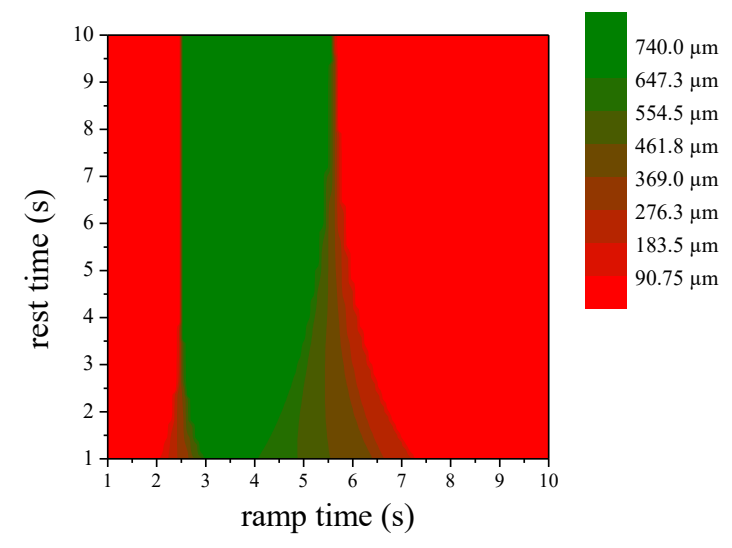

Fig. 2. (color online) Difference in final position of $10 \mu \mathrm{m}$ and $15 \mu \mathrm{m}$ polystyrene particles for various $t_{\text {ramp }}$ and $t_{\text {rest }}$ values after four cycles. A $4 \mathrm{MHz}$ transducer was used in water for simulations, therefore the maximum separation is four times half the wavelength, $740 \mu \mathrm{m}$. Red color corresponds to no separation and green denotes full separation.

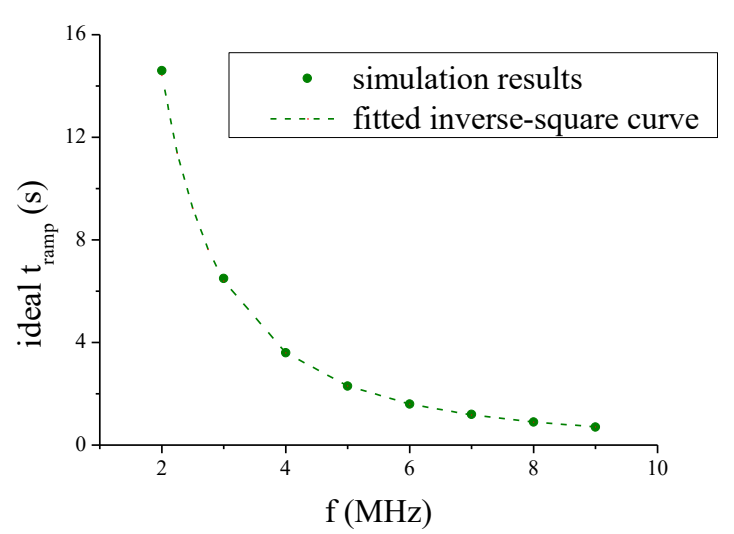

Fig. 3. Ideal ramp time vs frequency of transducer. The anticipated inverse square dependence has been verified by simulations.

The effect of frequency, acoustic pressure (transducer voltage) and density of media on ideal tramp time has been also investigated. Since increasing the frequency the acoustic force 
increases linearly and also the distance between nodes decreases in an inversely proportional manner, it was expected that the ideal tramp time is inversely proportional to the square of the transducer frequency. Simulation results revealed an almost perfect $f^{2}$ dependence as we anticipated (Fig. 3).

The acoustic radiation force has a quadratic dependence on the pressure amplitude, therefore again an inversely quadratic dependence of ideal $t_{\text {ramp }}$ on the pressure amplitude was expected and verified (Fig. 4). Since the pressure amplitude is directly proportional to the transducer voltage, the ideal $t_{\text {ramp }}$ is also proportional to $V^{-2}$.

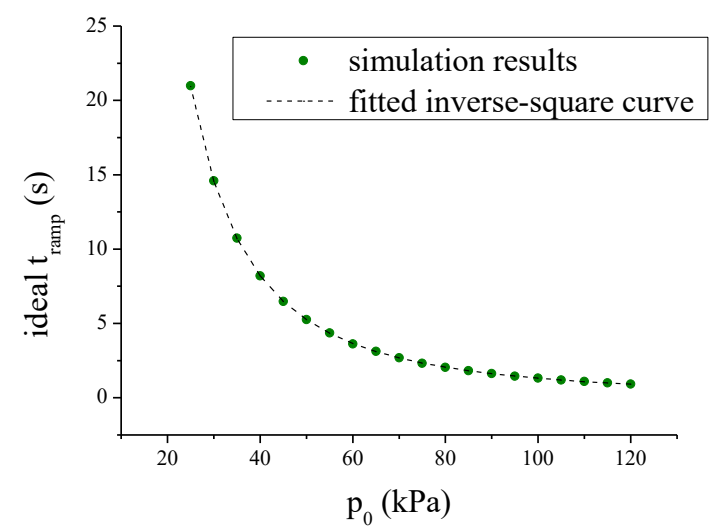

Fig. 4. Ideal ramp time vs the pressure amplitude (or transducer voltage). The anticipated inverse-square dependence has been verified by simulations.

Finally, the radiation force has a weak dependence on the density of the media, and therefore we predicted only a similarly weak dependence of ideal $t_{\text {ramp }}$ on the density which was again validated by the simulations (Fig. 5).

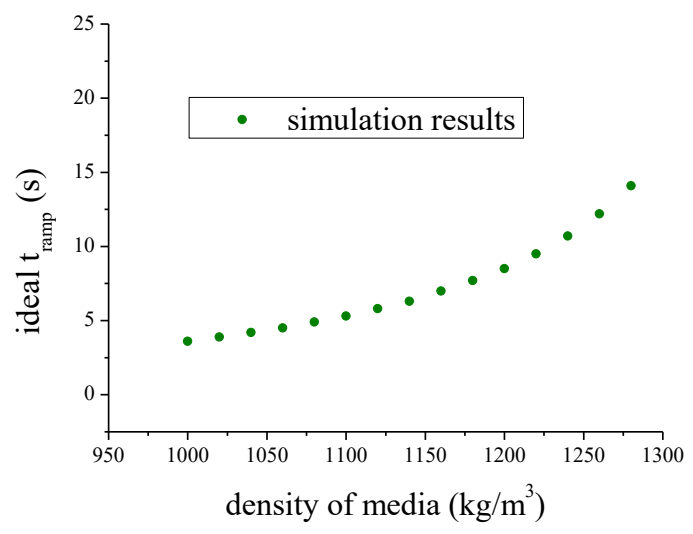

Fig. 5. Ideal ramp time vs the density of the media. Only a weak dependence can be observed.

These observations can help designing a device for separation of specific target cells or particles. The simulations also help choosing the appropriate field parameters for separation.

\section{B. Experimental Results}

To verify the equations of motion for particles presented in the theoretical section, two experiments have been carried out, one for size-based separation and one for density-based separation. For the experiments a previously described octagon bulk acoustic wave device was used [6]. To quantify the separation efficiency and purity we defined these as the following:

$$
\begin{gathered}
\text { Efficiency }=\frac{\text { number of target particles sorted }}{\text { number of all target particles }} \\
\text { Purity }=\frac{\text { number of target particles in sorted outlet }}{\text { all particles in sorted outlet }}
\end{gathered}
$$

For size-based separation 10 and $15 \mu$ m polystyrene particles have been suspended in aqueous solution. The applied voltage on the two PZT transducers was $10 \mathrm{Vpp}$, the frequency of the signal was $4 \mathrm{MHz}$. The experiments showed most effective separation for $2.5 \mathrm{~s}$ ramp time and $5 \mathrm{~s}$ rest time (Fig. 6). The recorded particle traces have been matched with theoretical results, the only fitting parameter being the acoustic pressure amplitude, $p_{0}=65 \mathrm{kPa}$. The theoretical and experimental results show excellent match (Fig. 7). For this experiment all of the large particles have been separated, resulting in $100 \%$ efficiency, and since some of the small particles also have been moved, the purity of the separation was $75 \%$.
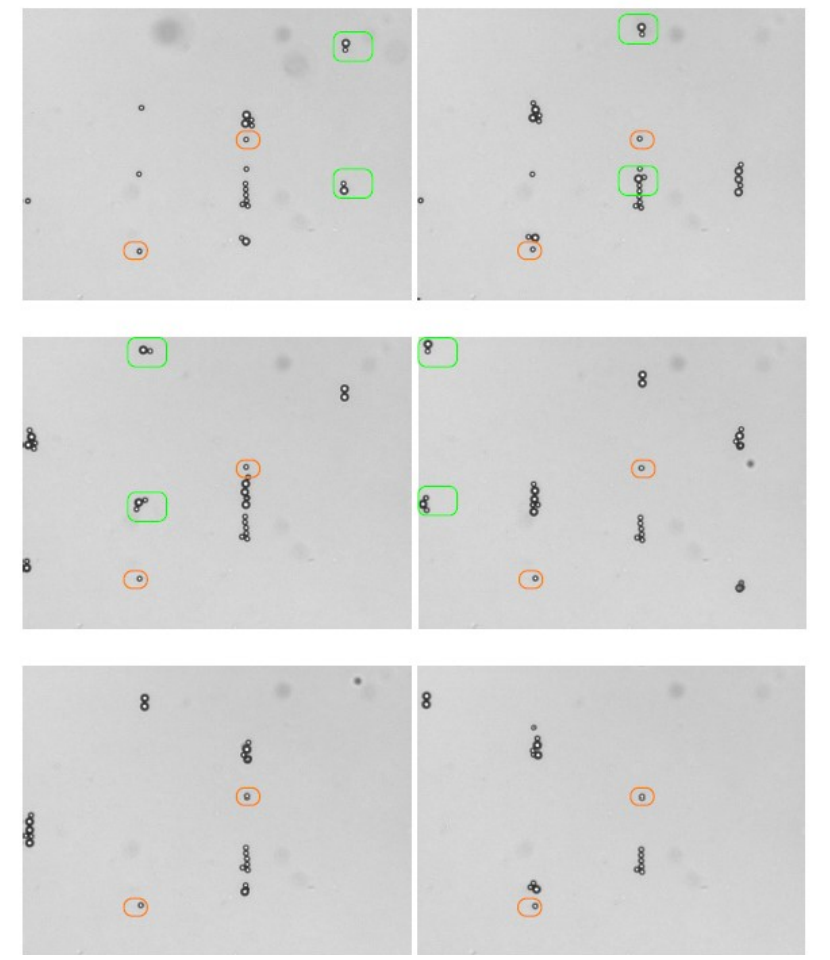

Fig. 6. (color online) Separation of $10 \mu \mathrm{m}$ and $15 \mu \mathrm{m}$ polystyrene particles. The images from left to right, top to bottom were recorded every 7.5 seconds (one after each full cycle). The large particles shift gradually to the left (in green) while the smaller ones (in orange) stay in place. 


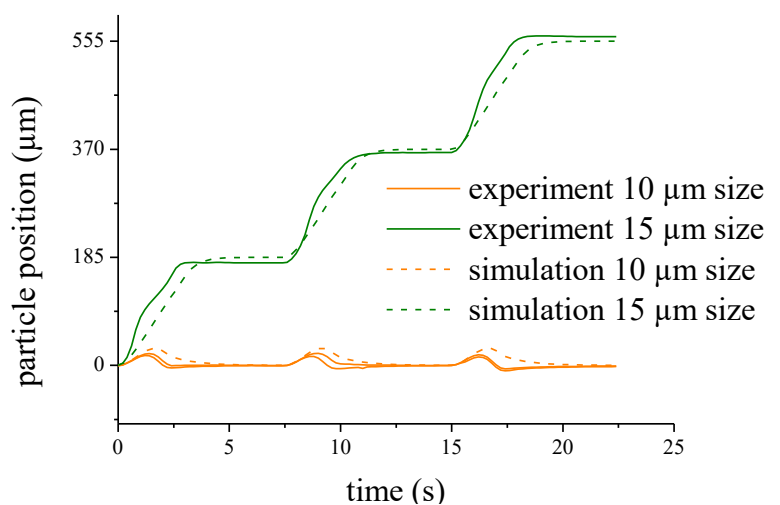

Fig. 7. Experimental and simulation results for size-based separation. An excellent fit can be observed for both types of particles.

For the density-based separation $10 \mu \mathrm{m}$ polystyrene $(\rho=$ $\left.1.05 \mathrm{~g} / \mathrm{cm}^{3}\right)$ and iron oxide $\left(\rho=1.7 \mathrm{~g} / \mathrm{cm}^{3}\right)$ particles have been used. The transducer voltage was reduced to $7 \mathrm{Vpp}$, the ramp time was $3 \mathrm{~s}$ and the rest time was $1.5 \mathrm{~s}$. Again a good fit between theoretical and experimental particle traces can be observed (Fig. 7). For this experiment the efficiency is $100 \%$ but the purity only $60 \%$.
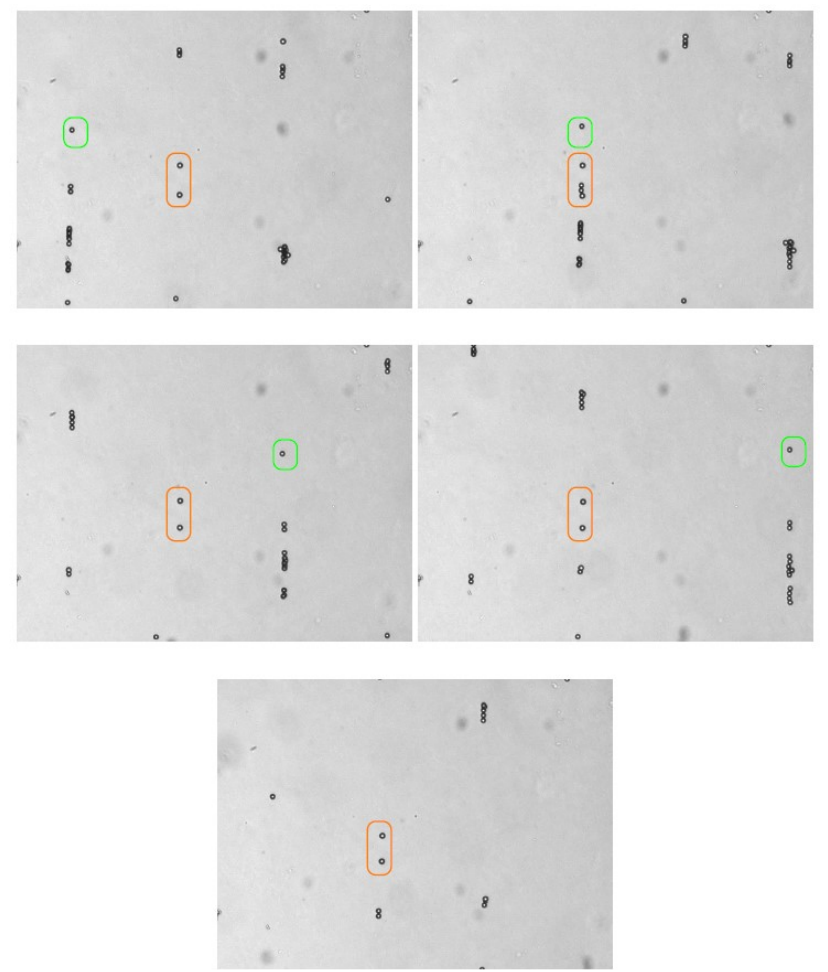

Fig. 8. (color online) Separation of $10 \mu \mathrm{m}$ polystyrene and iron oxide particles. The images from left to right, top to bottom were recorded every 4.5 seconds (one after each full cycle). The denser iron oxide particles shift gradually to the right (in green) while the less dense ones (in orange) stay in place.

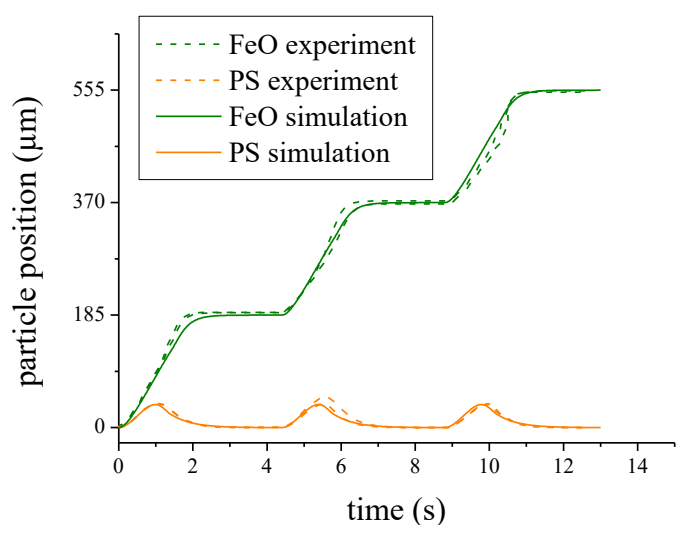

Fig. 9. Experimental and simulation results for density-based separation. An excellent fit can be observed for both types of particles.

\section{CONCLUSION}

The governing equations of the dynamic acoustic sorting have been presented in this paper. A numerical approach was introduced for determining the best field parameters for sorting. The effect of changing transducer voltage or frequency on sorting has been investigated along with changing the density of media. Experiments were carried out for size and density based separation, and the recorded particle traces were matched perfectly with simulation results. The efficiency of separation for both type of experiment was $100 \%$, but the purity of separation was lower for the density-based separation. This is due to the weak dependence of radiation force on density of particles. We shall investigate in the future the same principle of sorting for continuous flow devices.

\section{ACKNOWLEDGEMENT}

Part of this work was supported by Scottish Crucible and EPSRC From Molecules to Systems grants.

\section{REFERENCES}

[1] C. W. T. Shields, C. D. Reyes, and G. P. Lopez, "Microfluidic cell sorting: a review of the advances in the separation of cells from debulking to rare cell isolation," Lab Chip, vol. 15, pp. 1230-49, Mar 72015.

[2] F. Petersson, A. Nilsson, C. Holm, H. Jönsson, and T. Laurell, "Continuous separation of lipid particles from erythrocytes by means of laminar flow and acoustic standing wave forces," Lab Chip, vol. 5, pp. 20-22, 2005.

[3] B. L. Gray, H. Becker, Y. Ai, and B. L. Marrone, "Separation of biological cells in a microfluidic device using surface acoustic waves (SAWs)," in Proc. SPIE 8976, Microfluidics, BioMEMS, and Medical Microsystems XII, 89760O, 2014.

[4] D. A. Johnson and D. L. Feke, "Methodology for fractionating suspended particles using ultrasonic standing wave and divided flow fields," Separations Technology, vol. 5, pp. 251-258, 11// 1995.

[5] M. Wiklund, "Acoustofluidics 12: Biocompatibility and cell viability in microfluidic acoustic resonators," Lab Chip, vol. 12, pp. 2018-28, May 8 2012.

[6] G. D. Skotis, D. R. Cumming, J. N. Roberts, M. O. Riehle, and A. L. Bernassau, "Dynamic acoustic field activated cell separation (DAFACS)," Lab Chip, vol. 15, pp. 802-10, Feb 72015.

[7] H. Bruus, "Acoustofluidics 7: The acoustic radiation force on small particles," Lab Chip, vol. 12, pp. 1014-21, Mar 212012. 